\title{
Impact of the Malnutrition on Mortality in Elderly Patients Undergoing Percutaneous Coronary Intervention
}

\author{
Liling Chen, ', \\ Zhidong Huang, (D) ${ }^{2, *}$ Jin Lu, ',* \\ Yanfang Yang,' Yuxiong Pan,' \\ Kunming Bao,' Junjie Wang,' \\ Weihua Chen,' Jin Liu, ${ }^{2}$ \\ Yong Liu, ${ }^{2}$ Kaihong Chen, \\ Weiguo Li, 'Shiqun Chen ${ }^{2}$ \\ 'Department of Cardiology, Longyan \\ First Affiliated Hospital of Fujian Medical \\ University, Longyan, 364000, People's \\ Republic of China; ${ }^{2}$ Department of \\ Cardiology, Guangdong Provincial Key \\ Laboratory of Coronary Heart Disease \\ Prevention, Guangdong Cardiovascular \\ Institute, Guangdong Provincial People's \\ Hospital, Guangdong Academy of Medical \\ Sciences, Guangzhou, 510080, People's \\ Republic of China
}

*These authors contributed equally to this work

Correspondence: Shiqun Chen Department of Cardiology, Guangdong Provincial Key Laboratory of Coronary Heart Disease Prevention, Guangdong Cardiovascular Institute, Guangdong Provincial People's Hospital, Guangdong Academy of Medical Sciences, Guangzhou, 510080 , People's Republic of China

Tel +862083827812 ext. 10528

Fax +86 208385। 483

Email shiqunchen@I26.com

Weiguo Li

Department of Cardiology, Longyan First Affiliated Hospital of Fujian Medical

University, Longyan, 364000, People's

Republic of China

Tel +865973082105

Fax +865972100500

Emaillwg70120I@I63.com
Purpose: Malnutrition has been shown to be related to adverse clinical outcomes in patients with heart failure, hypertension, atrial fibrillation and other cardiovascular diseases. However, in the patients with coronary artery disease (CAD) undergoing percutaneous coronary interventions (PCI), especially in the elderly, the association of nutritional state and all-cause mortality remains unknown. We aimed to investigate the association of malnutrition with all-cause mortality in the elder patients undergoing PCI.

Patients and Methods: Based on the largest retrospective and observational cohort study from January 2007 to December 2017, the Controlling Nutritional Status (CONUT) score was applied to 21,479 consecutive patients with age $\geq 60$ who undergoing PCI for nutritional assessment. Participants were classified as absent, mild, moderate and severe malnutrition by CONUT score. The Kaplan-Meier method was used to compare all-cause mortality among the above four groups. Multivariable Cox proportional hazard regression analyses were performed to examine the association of malnutrition with all-cause mortality.

Results: According to the CONUT score, $48.19 \%, 15.08 \%$ and $0.94 \%$ patients were mildly, moderately and severely malnourished, respectively. During a median follow-up of 5.16 years (interquartile range: 3.02 to 7.89 years), 3173 (14.77\%) patients died. Kaplan-Meier analysis showed that the risk of all-cause mortality was significantly higher in patients with a worse nutritional status. Compared with normal nutritional state, malnutrition was associated with significantly increased risk for all-cause mortality (adjusted hazard ratio for mild, moderate and severe degrees of malnutrition, respectively: 1.20 [95\% confidence interval (CI): 1.09 to 1.33 ], 1.32 [95\% CI: 1.17 to 1.49 ] and 1.76 [95\% CI: 1.33 to 2.33 ]).

Conclusion: Malnutrition is prevalent among elderly patients with CAD undergoing PCI, and is strongly related to the all-cause mortality increasing. For elderly patients with CAD undergoing PCI, it is necessary to assess the status of nutrition, and evaluate the efficacy of nutritional interventions.

Keywords: malnutrition, elderly, percutaneous coronary intervention, Controlling Nutritional Status score

\section{Introduction}

Malnutrition is very common in the elderly patients, with a high prevalence range from $16 \%$ to $78 \%$ based on different nutritional assessment tools. ${ }^{1-5}$ These malnourished elderly will have higher complication and infection rates, longer duration of mean stay, costly medical expenditure, and increased mortality. ${ }^{6,7}$ With the progress of medical technology, more and more elderly people with coronary heart disease have undergone percutaneous coronary interventions (PCI). ${ }^{8,9}$ Such 
high-risk populations are more likely to be weaker and often combined with some basic diseases and leading to negative clinical outcomes. ${ }^{10,11}$ At present, no data are available on malnutrition-related morbidity and mortality in the elderly who underwent PCI.

High-risk patients should be identified based on modifiable clinical characteristics so that pre-emptive symptom management interventions can be initiated on these variables to improve their prognosis. Compared to other clinical variables, malnutrition is an important modifiable risk factor for poor clinical outcomes. ${ }^{12}$ Recently, several clinical studies have shown that poor nutritional status is associated with an increased risk of mortality in patients with cardiovascular disease, including acute heart failure, hypertension or atrial fibrillation, and few studies focus on the elderly patients with coronary artery disease (CAD) undergoing PCI. ${ }^{13-15}$

Therefore, the present study aimed to evaluate the impact of malnutrition on all-cause mortality among elderly patients with CAD undergoing PCI treatment.

\section{Patients and Methods}

\section{Study Population}

The Cardiorenal ImprovemeNt (CIN) study is a single-center, retrospective and observational cohort study, enrolling 43,415 consecutive patients undergoing PCI in Guangdong Provincial People's Hospital, Guangdong, China, hospitalized in between
January 2007 and December 2018 (ClinicalTrials.gov NCT04407936). PCI was performed following standard clinical practice guidelines. The patients younger than 60 years old $(n=16,192)$ or lack of data needed to assess malnutrition $(n=2093)$ were excluded from this analysis. We also excluded patients with missing data on follow-up ( $n=3651)$. Eventually, 21,479 patients were included (Figure 1).

All traceable personal identifiers were removed from the analytic dataset to protect patients' privacy. The study protocol was approved by Guangdong Provincial People's Hospital ethics committee and the study was performed according to the declaration of Helsinki [No. GDREC2019555H(R1)]. Informed consent was also not required for this study by the Guangdong Provincial People's Hospital Ethics Committee.

\section{Malnutrition Screening Tools}

The Controlling Nutritional Status (CONUT) score was determined by serum albumin, total cholesterol, and lymphocyte count, collected in the early morning after overnight fasting at admission (Table 1). ${ }^{16}$ A score of 0 to 1 is considered as absent; scores of 2 to 4,5 to 8 , and 9 to 12 reflect mild, moderate, and severe malnutrition, respectively. ${ }^{16}$

\section{Endpoint and Follow-Up}

The endpoint of our study was all-cause mortality. Demographic data, Laboratory data and medical therapy
Patients underwent percutaneous coronary at Guangdong
Provincial People's Hospital from January 2009 to
December $2018(\mathrm{n}=43415)$

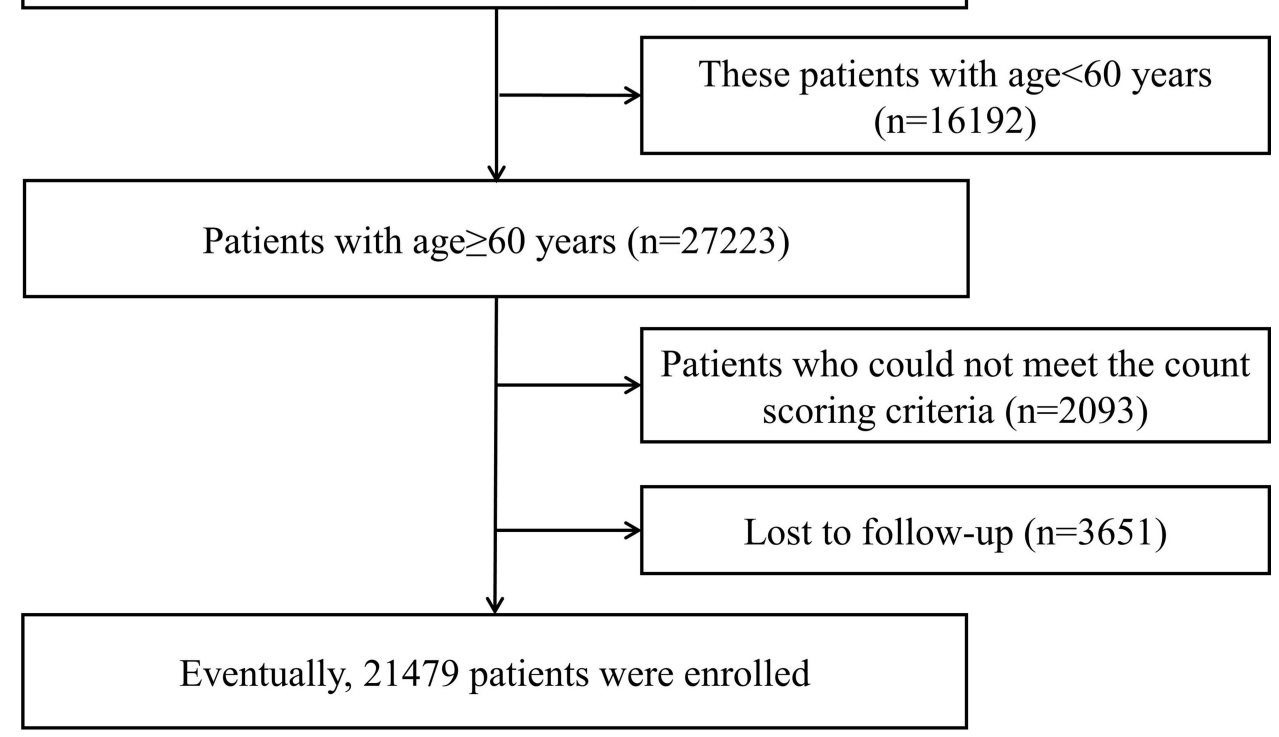

Figure I Patient flow diagram. 
Table I Malnutrition Screening Tools

\begin{tabular}{|c|c|c|c|c|c|}
\hline & & \multicolumn{4}{|c|}{ Risk of Malnutrition } \\
\hline & & Absent & Mild & Moderate & Severe \\
\hline CONUT, points & & $0-1$ & $2-4$ & $5-8$ & $9-12$ \\
\hline Formula & $\begin{array}{l}\text { Serum albumin, g/dl } \\
\text { Albumin score } \\
\text { Total cholesterol, mg/dl } \\
\text { Cholesterol score } \\
\text { Lymphocytes, count/mL } \\
\text { Lymphocytes score }\end{array}$ & $\begin{aligned} \geq & 3.50 \\
& 0 \\
\geq & 180 \\
& 0 \\
\geq & 1600 \\
& 0\end{aligned}$ & $\begin{array}{c}3.00-3.49 \\
2 \\
140-179 \\
1 \\
1200-1599 \\
1\end{array}$ & $\begin{array}{c}2.50-2.99 \\
4 \\
100-139 \\
2 \\
800-1199 \\
2\end{array}$ & $\begin{array}{c}<2.50 \\
6 \\
<100 \\
3 \\
<800 \\
3\end{array}$ \\
\hline
\end{tabular}

Abbreviation: CONUT, Controlling Nutritional Status.

data were collected from the electronic clinical management records system of the Guangdong Provincial People's Hospital from January 2009 to December 2018. Through the records system, we had access to all primary and secondary care records. During the study period, the information on whether he/she died and the date of death for each patient was retrieved from Guangdong Public Security System, which linked to CIN dataset by unique identified number.

Every person older than 60 years is considered elderly according to the definition of the World Health Organization (WHO). Low kidney function was defined as an estimated glomerular filtration rate (eGFR) $<60 \mathrm{~mL} /$ $\min / 1.73 \mathrm{~m}^{2}$. Pre-acute myocardial infarction was diagnosed according to the Fourth Universal Definition of Myocardial Infarction (2018). Anaemia was defined as a hematocrit $\leq 39 \%$ (male) or $\leq 36 \%$ (female). Acute myocardial infarction (AMI), diabetes mellitus (DM) and hypertension were defined by the 10th Revision Codes of the International Classification of Diseases.

\section{Statistical Analysis}

Continuous variables were expressed as the mean \pm standard deviation. Categorical variables were presented as frequencies and percentages. The Student's $t$ test was performed to test the normality of continuous variables. The comparison of categorical variables among groups was done by using a chi-square test. Time-to-event data were presented graphically using Kaplan-Meier curves. Log rank tests were used to compare survival between groups. A Cox proportional hazards regression analysis was conducted to determine whether there was a relationship between malnutrition and all-cause mortality. Various variables were included in the univariate and multivariate Cox proportional hazard analyses, such as age, gender, congestive heart failure, hypertension, atrial fibrillation, DM, eGFR $<60$, anemia, angiotensinconverting enzyme inhibitors or angiotensin receptor blockers, beta-blockers and statin. Adjusted multivariate variables included those variables associated with known poor prognosis based on clinical plausibility or $p$ value of $<0.05$ in the univariate Cox analyses.

\section{Results}

\section{Patients Characteristics}

Overall, 21,479 elderly patients undergoing PCI were enrolled, and those patients were divided into four groups according to the CONUT score. The percentage of malnutritional patients accounted for $64.21 \%(\mathrm{n}=13,792)$ and $48.19 \%, 15.08 \%$ and $0.94 \%$ patients were mildly, moderately and severely malnourished, respectively. Figure 2 shows the distribution of CONUT scores. The mean age was $69.53 \pm 6.59$ years, most patients were more likely to be male $(72.59 \%, \mathrm{n}=15,591), 1290$ had pre-acute myocardial infarction $(6.02 \%)$, the proportion of left ventricular ejection fraction (LVEF) $<40 \%$ was $9.77 \%(\mathrm{n}=1331)$. Patients with poor nutritional were older, were more likely to be man. They had a higher proportion of hypertension, DM, pre-myocardial infarction and eGFR $<60$. Additionally, evidence-based secondary prevention drugs, such as $\beta$-blockers and statin, were less commonly prescribed to those patients. More details of the baseline characteristics of patients enrolled are shown in Table 2.

\section{Nutritional Status and All-Cause Mortality}

During a median follow-up of 5.16 years (interquartile range: 3.02 to 7.89 years), 3173 (14.77\%) patients died. All-cause mortality among the total patients was $9.99 \%$ 


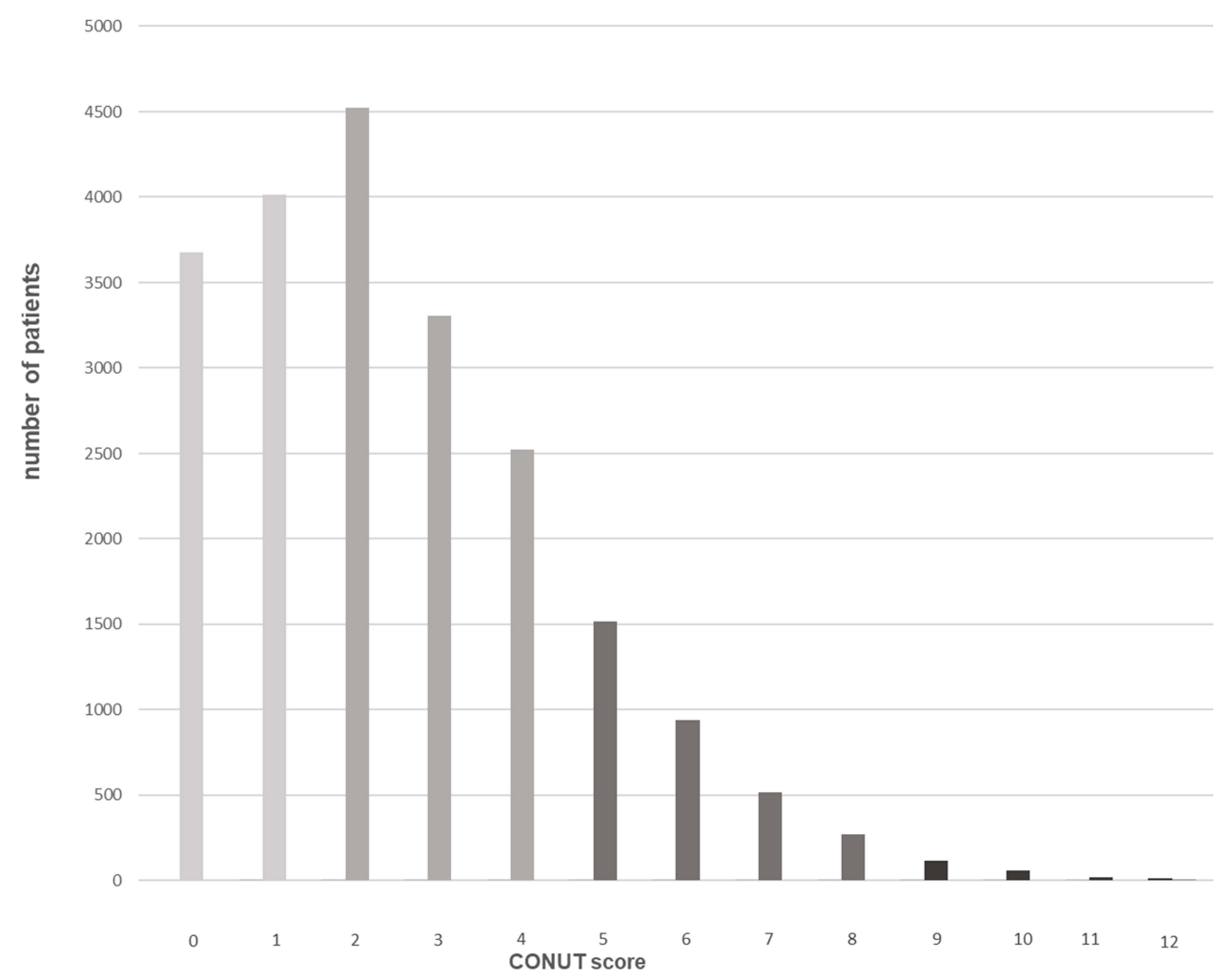

Figure 2 Distribution of CONUT scores. A score of 0 to $I$ is considered as absent; scores of 2 to 4,5 to 8 , and 9 to 12 reflect mild, moderate, and severe malnutrition, respectively.

Abbreviation: CONUT, Controlling Nutritional Status score.

(768), $15.32 \%$ (1586), $22.78 \%$ (738), $40.10 \%$ (81), respectively for four groups stratified by CONUT score.

As determined by Kaplan-Meier curves, the risk of allcause mortality increased in accordance with the severity of malnutrition $(\mathrm{p}<0.001)$ (Figure 3). The result indicated malnutrition was significantly associated with increased risk for all-cause mortality compared with normal nutritional state (adjusted hazard ratio for mild, moderate and severe degrees of malnutrition, respectively: 1.20 [95\% confidence interval (CI): 1.09 to 1.33], 1.32 [95\% CI: 1.17 to 1.49 ] and 1.76 [95\% CI: 1.33 to 2.33 ]) (Table 3).

Subgroup analysis showed that the patients with malnutrition were more likely to be related to the increased risk of all-cause death than absent patients among male or diabetes (Figure 4).

\section{Discussion}

To our knowledge, this was the first largest cohort realworld study in a developing country to explore the relationship between malnutrition and all-cause death in the elder patients who underwent PCI. In our study, we discovered malnutrition was widespread and predictive for all-cause mortality.
In our study, approximately two-thirds of the patients were malnourished, and nearly a fifth had moderate or severe undernutrition. A latest study showed that only 1/ 3 patients were malnourished by Geriatric Nutritional Risk Index (GNRI) in a cohort of 2853 patients with CAD who underwent PCI. ${ }^{17}$ A possible explanation for this could be that the present study focused on the older people. On the other hand, there are inconsistencies among various scoring tools for identifying the severity of malnutrition. ${ }^{18}$ The CONUT score used in our study was first reported by Ignacio de Uli'barri et al as an objective screening tool for identifying undernutrition in a hospital population. ${ }^{16}$ It seemed to be a simple malnutrition assessment tool to identify efficiently patients at risk of malnutrition. ${ }^{18,19}$ Furthermore, it can evaluate the protein reserve, calorie depletion, and immune defenses comprehensively. ${ }^{20}$ It should be highlighted that the older patients with PCI have more comorbid diseases, lower physiologic reserve, and more functional impairments (including physical capabilities and cognition), which may also contribute to the higher risk for post-AMI mortality. ${ }^{21}$ Consequently, our first suggestion is the need to screen for malnutrition in all patients admitted for CAD undergoing PCI. 
Table 2 Baseline Characteristic in the Elder Patients Undergoing PCI Stratified by CONUT Score

\begin{tabular}{|c|c|c|c|c|c|c|}
\hline Characteristics & Overall $n=21,479$ & Absent $n=7687$ & Mild $n=10,350$ & Moderate $n=3240$ & Severe $\mathrm{n}=202$ & P-value \\
\hline \multicolumn{7}{|c|}{ Demographic characteristic } \\
\hline Male, n (\%) & $|5,59|$ (72.59) & $5091(66.23)$ & $7766(75.03)$ & $2567(79.23)$ & $167(82.67)$ & $<0.001$ \\
\hline Age, years & $69.53 \pm 6.59$ & $68.15 \pm 6.06$ & $69.82 \pm 6.62$ & $71.66 \pm 6.95$ & $72.88 \pm 6.98$ & $<0.001$ \\
\hline \multicolumn{7}{|l|}{ Coexisting conditions } \\
\hline AMI, n (\%) & $4682(21.84)$ & $949(12.36)$ & $2266(21.94)$ & $\mid 356(4 \mid .92)$ & III (54.95) & $<0.001$ \\
\hline Hypertension, n (\%) & $13,488(62.90)$ & $4902(63.84)$ & 6495 (62.89) & $1958(60.53)$ & $133(65.84)$ & 0.009 \\
\hline Atrial fibrillation, n (\%) & $612(2.85)$ & I8I (2.35) & $299(2.89)$ & $123(3.80)$ & $9(4.46)$ & $<0.001$ \\
\hline Pre-MI, n (\%) & $1290(6.02)$ & $406(5.29)$ & $675(6.54)$ & $198(6.12)$ & II (5.45) & 0.006 \\
\hline DM, n (\%) & $6442(30.04)$ & $2242(29.20)$ & 3097 (29.99) & $1032(31.90)$ & 71 (35.15) & 0.015 \\
\hline eGFR< $60, \mathrm{n}(\%)$ & $5151(28.68)$ & $1280(20.73)$ & $2500(28.82)$ & $1238(42.44)$ & I33 (68.2I) & $<0.001$ \\
\hline Anemia, n (\%) & $8528(39.70)$ & $1872(24.35)$ & $4355(42.08)$ & $2139(66.02)$ & $162(80.20)$ & $<0.001$ \\
\hline CAD, n (\%) & $21,437(99.80)$ & 7667 (99.74) & $10,332(99.83)$ & $3236(99.88)$ & $202(100.00)$ & 0.369 \\
\hline CHF, n (\%) & $2356(10.98)$ & $528(6.87)$ & $1099(10.63)$ & $658(20.36)$ & 7I (35.15) & $<0.001$ \\
\hline LVEF<40\%, n (\%) & $|33|(9.77)$ & $324(6.51)$ & $619(9.55)$ & $354(17.40)$ & $34(27.42)$ & $<0.001$ \\
\hline \multicolumn{7}{|l|}{ Laboratory examination } \\
\hline WBC, $10^{9} / \mathrm{L}$ & $8.00 \pm 2.78$ & $7.83 \pm 2.23$ & $7.90 \pm 2.72$ & $8.62 \pm 3.71$ & $9.84 \pm 4.50$ & $<0.001$ \\
\hline Hemoglobin, g/L & $129.90 \pm 16.68$ & $135.10 \pm 14.20$ & $|29.67 \pm| 5.7 \mid$ & $119.83 \pm 18.57$ & $105.55 \pm 21.64$ & $<0.001$ \\
\hline LYM, $10^{9} / \mathrm{L}$ & $1.8 I \pm 0.67$ & $2.14 \pm 0.60$ & $1.75 \pm 0.63$ & $1.28 \pm 0.54$ & $0.87 \pm 0.36$ & $<0.001$ \\
\hline Total cholesterol, $\mathrm{mmol} / \mathrm{L}$ & $4.49 \pm 1.17$ & $5.09 \pm 1.07$ & $4.26 \pm 1.08$ & $3.88 \pm 1.06$ & $3.29 \pm 0.83$ & $<0.001$ \\
\hline Albumin, $g / L$ & $35.60 \pm 4.23$ & $38.5 I \pm 2.56$ & $35.29 \pm 3.36$ & $30.38 \pm 3.34$ & $24.63 \pm 3.09$ & $<0.001$ \\
\hline eGFR, $\mathrm{mL} / \mathrm{min} / 1.73 \mathrm{~m}^{2}$ & $72.27 \pm 24.06$ & $76.76 \pm 21.99$ & $72.25 \pm 23.43$ & $64.39 \pm 26.74$ & $48.95 \pm 28.20$ & $<0.001$ \\
\hline \multicolumn{7}{|l|}{ Medications } \\
\hline ACEI or ARB, $n(\%)$ & $10,776(51.03)$ & $3863(50.58)$ & $5257(51.57)$ & $1572(50.55)$ & $84(47.46)$ & 0.389 \\
\hline Beta-blockers, n (\%) & $|7,44|$ (82.59) & $6366(83.36)$ & $8443(82.83)$ & $2501(80.42)$ & $|3|(74.01)$ & $<0.001$ \\
\hline CCB, n (\%) & $5003(23.69)$ & $1963(25.70)$ & $2382(23.37)$ & 615 (19.77) & $43(24.29)$ & $<0.001$ \\
\hline Statin, n (\%) & $20,629(97.69)$ & 7501 (98.22) & 9957 (97.68) & 3007 (96.69) & $164(92.66)$ & $<0.001$ \\
\hline
\end{tabular}

Note: Values are means \pm SDs or $\mathrm{n}(\%)$.

Abbreviations: AMI, acute myocardial infarction; Pre-MI, pre-myocardial infarction; DM, diabetes mellitus; CAD, coronary artery disease; CHF, congestive heart failure; LVEF, left ventricular ejection fraction; WBC, white blood cell; LYM, lymphocyte; eGFR, estimated glomerular filtration rate; ACEl, angiotensin-converting enzyme inhibitors; $\mathrm{ARB}$, angiotensin receptor blockers; $\mathrm{CCB}$, calcium blocker.

This study demonstrated that malnutrition was associated with poor prognosis in patients older than 60 years who underwent PCI. We found that, regardless of clinical variables, coronary revascularization, and optimal medical treatment, malnutrition was still associated with all-cause mortality. The issue deserves to remark is that the optimal medications can both improve the prognosis of myocardial infarction in the univariate analysis and the Cox multivariate analysis. Though there is no significant association between the use of Beta-blockers and all-cause mortality in the Cox multivariate analysis, it still showed similar protective effects in the Cox multivariate analysis as other medications. The benefit of long-term treatment with oral beta-blockers after AMI is well established, though one study including 6758 patients with previous MI, beta- blocker use was not associated with a lower risk of cardiovascular events or mortality. ${ }^{22-24}$ Afterall, we believe that oral beta-blocker initiation should be considered within the first $24 \mathrm{~h}$ in haemodynamically stable patients who underwent PCI. The above candidate variables were based on the literatures and clinical relevance. Recently, there has been an increasing research interest in the relationship between malnutrition and the prognosis of patients undergoing PCI by various nutritional assessments. ${ }^{17,25-27}$ These findings similarly demonstrated that patients with malnutrition had worse clinical outcomes. Wada et al showed a significant association between malnutrition, based on Combined Objective Nutritional Score [determined by assigning 1 point each for high CONUT score, low GNRI $(<98)$ or low Prognostic Nutritional Index score 


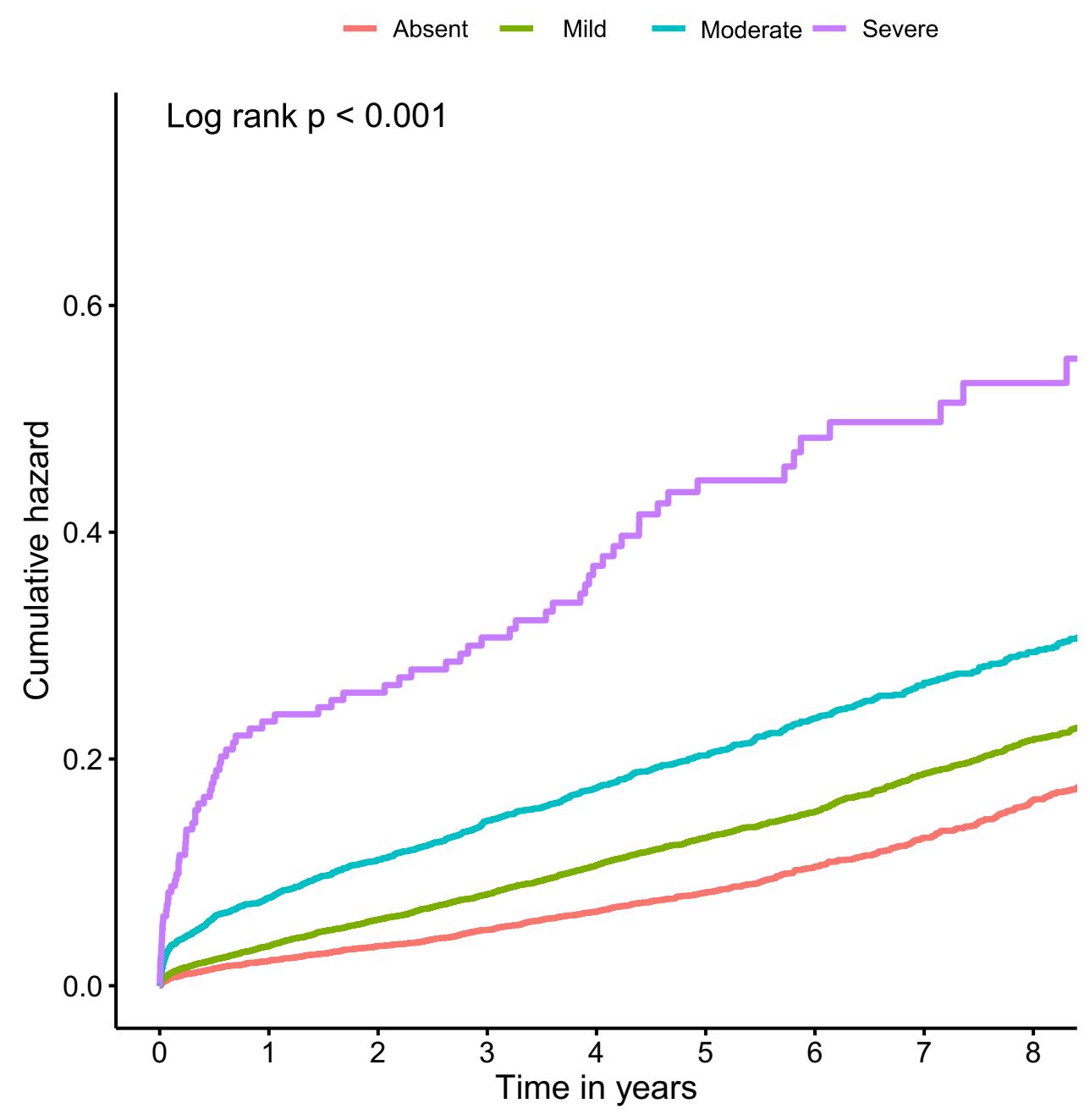

Figure 3 Kaplan-Meier curves for all-cause mortality by the CONUT score. A score of 0 to I is considered as absent; scores of 2 to 4,5 to 8 , and 9 to 12 reflect mild, moderate, and severe malnutrition, respectively.

Abbreviation: CONUT, Controlling Nutritional Status score.

(PNI) $(<45)$ ], and all-cause death and cardiac death in a cohort of 1984 patients with CAD who have undergone PCI. ${ }^{5,11-16,28-31}$ Additionally, Raposeiras Roubín et al also indicated that malnutrition [assessed by CONUT score, PNI and Nutritional Risk Index (NRI)] was strongly associated with increased mortality and cardiovascular events in 5062 consecutive patients with acute coronary syndrome (ACS) ${ }^{18}$ Chen et al showed that CONUT score has been associated with a higher incidence of major acute cardiovascular events (MACE) in patients with ACS undergoing PCI. ${ }^{32}$ Basta et al also found that patients at severe malnutrition had a significantly higher risk of events for all-cause death than those with no malnutrition by the CONUT score and PNI in a cohort of 945 patients with acute ST-Segment elevation myocardial infarction undergoing primary percutaneous coronary intervention with stent. ${ }^{33}$ These studies were relatively small, and larger studies are required to confirm these findings. Furthermore, our population was composed predominantly of the elders undergoing elective or emergent PCI, who were more likely to be associated with a high incidence of adverse outcomes after undergoing PCI. ${ }^{29,30}$

The possible underlying mechanism linking malnutrition to all-cause mortality may be that the activation of inflammatory pathways plays an important role. Some studies have reported an association between higher level of inflammation, worse nutritional status, and adverse clinical outcomes in patients with congestive heart failure (CHF) ${ }^{20,34}$ Mechanistically, the activation of neurohormonal and inflammatory pathways that characterize cardiovascular disease may increase the catabolic demand, and patients with already poor nutritional status may be more 
Table 3 Univariate and Multivariate Cox Proportional Hazards Model for the Association Between Malnutrition and All-Cause Death

\begin{tabular}{|c|c|c|c|c|}
\hline & \multicolumn{2}{|c|}{ Univariate Analysis } & \multicolumn{2}{|c|}{ Multivariable Analysis } \\
\hline & HR $[95 \% \mathrm{Cl}]$ & p-value & $\mathrm{HR}[95 \% \mathrm{Cl}]$ & p-value \\
\hline Continuous CONUT score & $1.15(1.14-1.17)$ & $<0.001$ & $1.06(1.04-1.08)$ & $<0.001$ \\
\hline \multicolumn{5}{|l|}{ Malnutrition, degree } \\
\hline Absent & Ref & & Ref & \\
\hline Mild & $1.46(1.34-1.59)$ & $<0.001$ & $1.20(1.09-1.33)$ & $<0.001$ \\
\hline Moderate & $2.08(1.88-2.30)$ & $<0.001$ & $1.32(1.17-1.49)$ & $<0.001$ \\
\hline Severe & $4.16(3.3 I-5.23)$ & $<0.001$ & $1.76(1.33-2.33)$ & $<0.001$ \\
\hline Atrial fibrillation & $1.90(1.62-2.24)$ & $<0.001$ & $1.41(1.17-1.69)$ & $<0.001$ \\
\hline DM & $1.29(1.20-1.39)$ & $<0.001$ & $1.20(1.10-1.30)$ & $<0.001$ \\
\hline eGFR $<60$ & $2.04(1.89-2.20)$ & $<0.001$ & $1.44(1.32-1.56)$ & $<0.001$ \\
\hline Anemia & $1.45(1.36-1.56)$ & $<0.001$ & $1.12(1.03-1.21)$ & 0.003 \\
\hline $\mathrm{CHF}$ & $2.50(2.28-2.74)$ & $<0.001$ & $1.75(1.57-1.94)$ & $<0.001$ \\
\hline ACEI or ARB & $0.81(0.76-0.88)$ & $<0.001$ & $0.86(0.80-0.93)$ & $<0.001$ \\
\hline Beta-blockers & $0.80(0.73-0.87)$ & $<0.001$ & $0.92(0.83-1.02)$ & 0.096 \\
\hline Statin & $0.58(0.48-0.70)$ & $<0.001$ & $0.73(0.60-0.89)$ & 0.002 \\
\hline
\end{tabular}

Notes: Adjusted by gender, age, hypertension, atrial fibrillation, diabetes mellitus, eGFR< 60 , chronic kidney disease, anaemia, congestive and heart failure, angiotensinconverting enzyme inhibitors or angiotensin receptor blockers, beta-blockers, statin.

Abbreviations: $\mathrm{Cl}$, confidence interval; HR, hazard ratio; $\mathrm{AMI}$, acute myocardial infarction; $\mathrm{CAD}$, coronary artery disease; $\mathrm{CHF}$, congestive heart failure; Pre-MI, Premyocardial infarction; DM, diabetes mellitus; LVEF, left ventricular ejection fraction; WBC, white blood cell; LYM, lymphocyte; eGFR, estimated glomerular filtration rate; ACEI, angiotensin-converting enzyme inhibitors; ARB, angiotensin receptor blockers; CCB, calcium blocker; CONUT, Controlling Nutritional Status.

vulnerable to cardiac events. Tumor necrosis factor-alpha (TNF- $\alpha$ ), one of the key cytokines mediating inflammation and promoting catabolism, was found to be significantly higher in the patients with moderate or severe malnutrition. ${ }^{20,35}$ Another study also confirmed that hypoalbuminemia, suggesting poor nutritional state, is associated with cardiac cachexia and extremely poor prognosis. $^{36}$

All those findings strongly support the need for physicians to practice early identification of malnutrition, risk stratification and clinical intensive managements, especially in the elderly patients undergoing PCI. Clinicians should stay abreast of the current scientific evidence to provide the most meaningful and effective nutrition guidance, aiming to individualize the nutritional support. A secondary prevention program that combines the nutritional intervention, a supervised exercise program, psychological treatment, advice to quit smoking and drug prescription should be encouraged to promote the Cardiac rehabilitation and physical fitness. Malnutrition is a state resulting from lack of uptake or intake of nutrition leading to altered body composition that requires long-term care and management. ${ }^{37}$ Some studies have shown that the use of targeted oral nutritional supplement strategies in older adults may reduce complications, mortality, and hospital readmissions. $^{38}$ Nutritional intervention may prevent complications and improve the quality of life in CHF patients. ${ }^{39,40}$ Consequently, clinical trials are needed to prospectively evaluate the efficacy of nutritional interventions on outcomes in old patients with CAD undergoing PCI and the optimal nutritional intervention therapy on the outcome in this population should be ensured.

Our research has some strengths. We focused on the elderly and frailer patients who need more attention from clinicians. Furthermore, our study population was the largest scale in the world and could be more epidemiologically representative of the elder population with CAD undergoing PCI. However, we must acknowledge some limitations. Firstly, this study is a single-center retrospective study, but the enrolled patients came from a big PCI center, which was representative and convincing in the sample and the study quality control. Secondly, the generalizability of our results is limited to the Chinese population without the consideration of the other races, but our findings could be extrapolated to other countries with health systems and populations similar to ours. Thirdly, we did not apply different nutritional screening tools and compare their prognostic value of malnutrition for allcause mortality. However, the CONUT score has been confirmed as the best tool to evaluate the nutritional status in the appliance on the patients with CAD. Fourth, other clinical outcome events such as cardiac vascular death, 


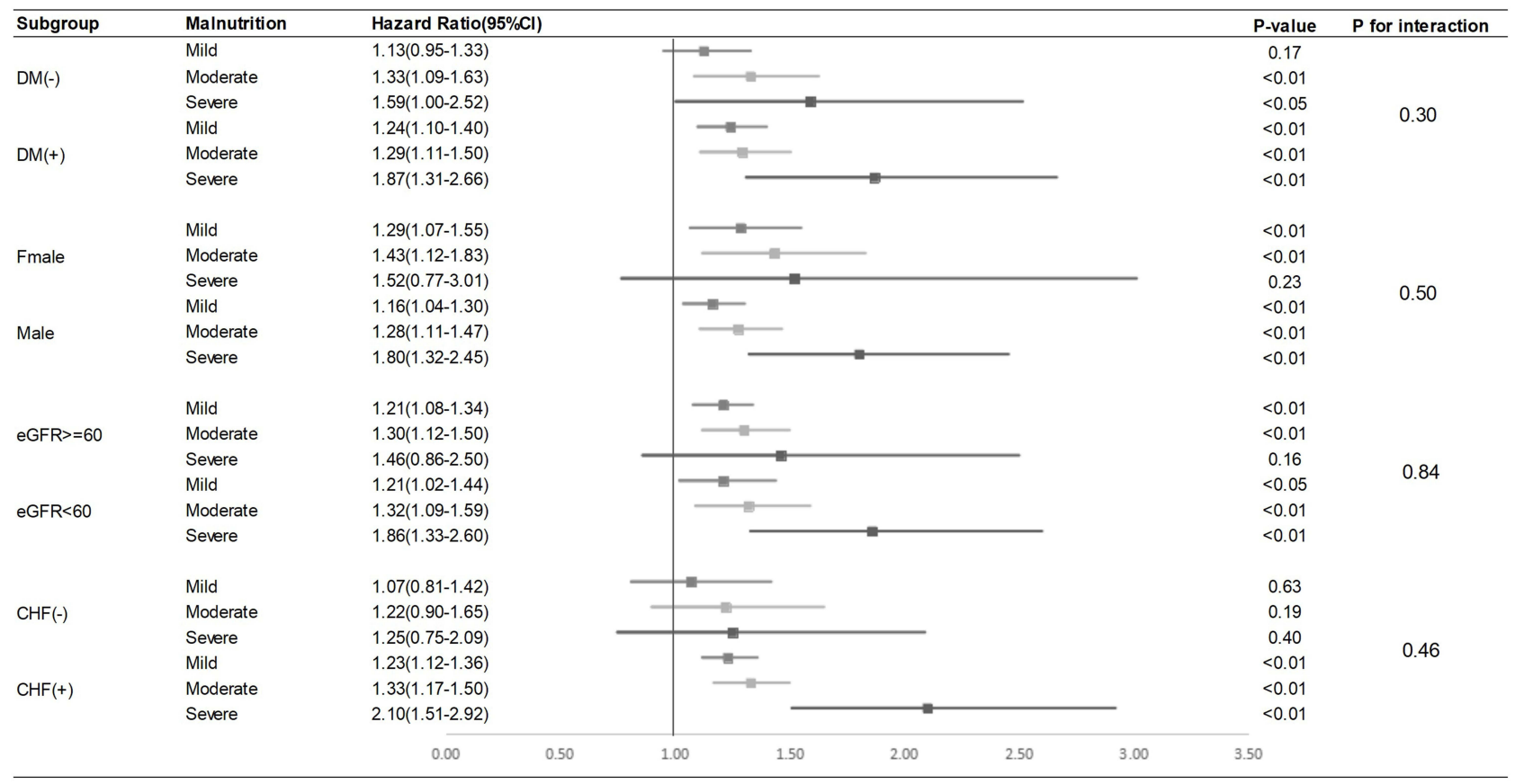

Figure 4 Forest plots of hazard ratios for the primary endpoint in different subgroups. Adjusted by age, gender, anemia, acute myocardial infarction, angiotensin-converting enzyme inhibitors or angiotensin receptor blockers, beta-blockers, chronic kidney disease, congestive heart failure, diabetes mellitus, diabetes mellitus, hypertension, statins, atrial fibrillation.

Abbreviations: DM (-), no diabetes mellitus, DM (+), diabetes mellitus; CHF (-), no congestive heart failure, CHF (+), congestive heart failure; DM, diabetes mellitus; Cl, confidence interval; CONUT, Controlling Nutritional Status score.

reinfarction, or ischemic stroke were not recorded, but primary point of our study was all-cause death, which was the most representative clinical end point for prognosis. Lastly, the nutritional evaluation was conducted only at baseline in hospitalization, we did not investigate the changes in nutritional status over time and their relationship with mortality.

\section{Conclusion}

Malnutrition is common status among elderly patients with CAD undergoing PCI, and is significantly associated with increased mortality. Physicians should stay abreast of the current scientific evidence to provide the most meaningful and effective nutrition guidance and practice early identification of malnutrition, risk stratification and clinical intensive managements. Study is needed to prospectively evaluate the efficacy of nutritional interventions on outcomes among these patients.

\section{Author Contributions}

The authors' responsibilities were as follows - Conception and study design: SQC, WGL, LLC, YL, and KHC; execution: LLC; acquisition of data: JLU, JLI, ZDH, YFY, YXP, KMB, JJW, WHC and KHC; analysis and interpretation: JLI, ZDH, JLU, YFY; drafting, revising or critically reviewing the article: SQC, LLC, WGL, ZDH, JLU, YFY. All authors made a significant contribution to the work reported, whether that is in the conception, study design, execution, acquisition of data, analysis and interpretation, or in all these areas; took part in drafting, revising or critically reviewing the article; gave final approval of the version to be published; have agreed on the journal to which the article has been submitted; and agree to be accountable for all aspects of the work. All authors read and approved the final version.

\section{Funding}

This study was supported by grants from Beijing Lisheng Cardiovascular Health Foundation and Guangdong Provincial People's Hospital Foundation (LHJJ20141751, DFJH201919) and National Science Foundation of China (81670339, 81970311), Natural Science Foundation of Fujian Provincial Science and Technology Department (2019J01617). The work was not funded by any industry sponsors.

\section{Disclosure}

The authors report no conflict of interest in this work. 


\section{References}

1. Corish CA, Kennedy NP. Protein-energy undernutrition in hospital in-patients. $\quad B r \quad J \quad$ Nutr. 2000;83(6):575-591. doi:10.1017/ S000711450000074X

2. Chern CJH, Lee S-D. Malnutrition in hospitalized Asian seniors: an issue that calls for action. J Clin Gerontol Geriatr. 2015;6(3):73-77. doi:10.1016/j.jcgg.2015.02.007

3. Stratton RJ, King CL, Stroud MA, Jackson AA, Elia M. 'Malnutrition universal screening tool' predicts mortality and length of hospital stay in acutely ill elderly. Br J Nutr. 2006;95(2):325-330. doi:10.1079/BJN20051622

4. Kaiser MJ, Bauer JM, Rämsch C, et al. Frequency of malnutrition in older adults: a multinational perspective using the mini nutritional assessment. J Am Geriatr Soc. 2010;58(9):1734-1738. doi:10.1111/ j.1532-5415.2010.03016.x

5. Bonetti L, Terzoni S, Lusignani M, Negri M, Froldi M, Destrebecq A. Prevalence of malnutrition among older people in medical and surgical wards in hospital and quality of nutritional care: a multicenter, cross-sectional study. J Clin Nurs. 2017;26(2324):5082-5092. doi:10.1111/jocn.14051

6. Shpata V, Ohri I, Nurka T, Prendushi X. The prevalence and consequences of malnutrition risk in elderly Albanian intensive care unit patients. Clin Interv Aging. 2015;10:481-486. doi:10.2147/CIA. S77042

7. Ljungqvist O, van Gossum A, Sanz ML, de Man F. The European fight against malnutrition. Clin Nutr. 2010;29(2):149-150. doi:10.1016/j.clnu.2009.10.004

8. Bradley SM, Bohn CM, Malenka DJ, et al. Temporal trends in percutaneous coronary intervention appropriateness: insights from the clinical outcomes assessment program. Circulation. 2015;132 (1):20-26. doi:10.1161/CIRCULATIONAHA.114.015156

9. Morita Y, Hamaguchi T, Yamaji Y, et al. Temporal trends in prevalence and outcomes of atrial fibrillation in patients undergoing percutaneous coronary intervention. Clin Cardiol. 2020;43(1):33-42. doi:10.1002/clc. 23285

10. Chen PF, Wang DN, Chen K, et al. Outcomes of percutaneous coronary intervention in patients $\geq 75$ years: one-center study in a Chinese patient group. J Geriatr Cardiol. 2015;12(6):626-633. doi:10.11909/j.issn.1671-5411.2015.06.004

11. Numasawa Y, Inohara T, Ishii H, et al. Comparison of outcomes after percutaneous coronary intervention in elderly patients, including 10 628 nonagenarians: insights from a Japanese Nationwide registry (J-PCI registry). $J$ Am Heart Assoc. 2019;8(5):e011183. doi:10.1161/JAHA.118.011017

12. Freeman AM, Morris PB, Barnard N, et al. Trending cardiovascular nutrition controversies. J Am Coll Cardiol. 2017;69(9):1172-1187. doi:10.1016/j.jacc.2016.10.086

13. Iwakami N, Nagai T, Furukawa TA, et al. Prognostic value of malnutrition assessed by controlling nutritional status score for long-term mortality in patients with acute heart failure. Int $J$ Cardiol. 2017;230:529-536. doi:10.1016/j.ijcard.2016.12.064

14. Sun X, Luo L, Zhao X, Ye P. Controlling nutritional status (CONUT) score as a predictor of all-cause mortality in elderly hypertensive patients: a prospective follow-up study. BMJ Open. 2017;7(9): e015649. doi:10.1136/bmjopen-2016-015649

15. Anaszewicz M, Budzyński J. Clinical significance of nutritional status in patients with atrial fibrillation: an overview of current evidence. J Cardiol. 2017;69(5):719-730. doi:10.1016/j.jjcc.2016.06.014

16. Ignacio de Ulíbarri J, González-Madroño A, de Villar NG, et al. CONUT: a tool for controlling nutritional status. First validation in a hospital population. Nutr Hosp. 2005;20(1):38-45.

17. Wada H, Dohi T, Miyauchi K, et al. Prognostic impact of the geriatric nutritional risk index on long-term outcomes in patients who underwent percutaneous coronary intervention. Am J Cardiol. 2017;119 (11):1740-1745. doi:10.1016/j.amjcard.2017.02.051
18. Raposeiras Roubín S, Abu Assi E, Cespón Fernandez M, et al. Prevalence and prognostic significance of malnutrition in patients with acute coronary syndrome. $J$ Am Coll Cardiol. 2020;76 (7):828-840. doi:10.1016/j.jacc.2020.06.058

19. Tonet E, Campo G, Maietti E, et al. Nutritional status and all-cause mortality in older adults with acute coronary syndrome. Clin Nutr. 2020;39(5):1572-1579. doi:10.1016/j.clnu.2019.06.025

20. Nakagomi A, Kohashi K, Morisawa T, et al. Nutritional status is associated with inflammation and predicts a poor outcome in patients with Chronic Heart Failure. J Atheroscler Thromb. 2016;23 (6):713-727. doi: $10.5551 /$ jat.31526

21. Dodson JA, Hajduk AM, Geda M, et al. Predicting 6-month mortality for older adults hospitalized with acute myocardial infarction: a Cohort Study. Ann Intern Med. 2020;172(1):12-21. doi:10.7326/ M19-0974

22. Freemantle N, Cleland J, Young P, Mason J, Harrison J. Beta Blockade after myocardial infarction: systematic review and meta regression analysis. BMJ. 1999;318(7200):1730-1737. doi:10.1136/ bmj.318.7200.1730

23. Goldberger JJ, Bonow RO, Cuffe M, et al. Effect of beta-blocker dose on survival after acute myocardial infarction. $J$ Am Coll Cardiol. 2015;66(13):1431-1441. doi:10.1016/j.jacc.2015.07.047

24. Bangalore $S$, Steg G, Deedwania $P$, et al. $\beta$-Blocker use and clinical outcomes in stable outpatients with and without coronary artery disease. JAMA. 2012;308(13):1340-1349. doi:10.1001/ jama.2012.12559

25. Chen QJ, Qu HJ, Li DZ, et al. Prognostic nutritional index predicts clinical outcome in patients with acute ST-segment elevation myocardial infarction undergoing primary percutaneous coronary intervention. Sci Rep. 2017;7(1):3285. doi:10.1038/s41598-01703364-x

26. Kunimura A, Ishii $\mathrm{H}$, Uetani $\mathrm{T}$, et al. Impact of nutritional assessment and body mass index on cardiovascular outcomes in patients with stable coronary artery disease. Int J Cardiol. 2017;230:653-658. doi:10.1016/j.ijcard.2017.01.008

27. Wada H, Dohi T, Miyauchi K, et al. Prognostic impact of nutritional status assessed by the controlling nutritional status score in patients with stable coronary artery disease undergoing percutaneous coronary intervention. Clin Res Cardiol. 2017;106(11):875-883. doi:10.1007/ s00392-017-1132-z

28. Wolters M, Volkert D, Streicher M, et al. Prevalence of malnutrition using harmonized definitions in older adults from different settings a MaNuEL study. Clin Nutr. 2019;38(5):2389-2398. doi:10.1016/j. clnu.2018.10.020

29. Bainey KR, Alemayehu W, Armstrong PW, Westerhout CM, Kaul P, Welsh RC. Long-term outcomes of complete revascularization with percutaneous coronary intervention in acute coronary syndromes. JACC Cardiovasc Interv. 2020;13(13):1557-1567. doi:10.1016/j. jcin.2020.04.034

30. McNeely C, Markwell S, Vassileva CM. Readmission after inpatient percutaneous coronary intervention in the Medicare population from 2000 to 2012. Am Heart J. 2016;179:195-203. doi:10.1016/j. ahj.2016.07.002

31. Wada H, Dohi T, Miyauchi K, et al. Combined effect of nutritional status on long-term outcomes in patients with coronary artery disease undergoing percutaneous coronary intervention. Heart Vessels. 2018;33(12):1445-1452. doi:10.1007/s00380-018-1201-x

32. Chen SC, Yang YL, Wu CH, et al. Association between preoperative nutritional status and clinical outcomes of patients with coronary artery disease undergoing percutaneous coronary intervention. Nutrients. 2020;12(5):1295. doi:10.3390/nu12051295

33. Basta G, Chatzianagnostou K, Paradossi U, et al. The prognostic impact of objective nutritional indices in elderly patients with ST-elevation myocardial infarction undergoing primary coronary intervention. Int $J$ Cardiol. 2016;221:987-992. doi:10.1016/j. ijcard.2016.07.039 
34. Izawa KP, Watanabe S, Hirano Y, et al. The relation between geriatric nutritional risk index and muscle mass, muscle strength, and exercise capacity in chronic heart failure patients. Int J Cardiol. 2014;177 (3):1140-1141. doi:10.1016/j.ijcard.2014.08.045

35. Springer J, Filippatos G, Akashi YJ, Anker SD. Prognosis and therapy approaches of cardiac cachexia. Curr Opin Cardiol. 2006;21 (3):229-233. doi:10.1097/01.hco.0000221585.94490.09

36. Pasini E, Aquilani R, Gheorghiade M, Dioguardi FS. Malnutrition, muscle wasting and cachexia in chronic heart failure: the nutritional approach. Ital Heart J. 2003;4(4):232-235.

37. Cederholm T, Bosaeus I, Barazzoni R, et al. Diagnostic criteria for malnutrition - an ESPEN consensus statement. Clin Nutr. 2015;34 (3):335-340. doi:10.1016/j.clnu.2015.03.001
38. Philipson TJ, Snider JT, Lakdawalla DN, Stryckman B, Goldman DP. Impact of oral nutritional supplementation on hospital outcomes. $\mathrm{Am}$ J Manag Care. 2013;19(2):121-128.

39. Otaki M. Surgical treatment of patients with cardiac cachexia. An analysis of factors affecting operative mortality. Chest. 1994;105 (5):1347-1351. doi:10.1378/chest.105.5.1347

40. Tevik K, Thürmer H, Husby MI, de Soysa AK, Helvik AS. Nutritional risk screening in hospitalized patients with heart failure. Clin Nutr. 2015;34(2):257-264. doi:10.1016/j.clnu.2014.03.014

\section{Publish your work in this journal}

Clinical Interventions in Aging is an international, peer-reviewed journal focusing on evidence-based reports on the value or lack thereof of treatments intended to prevent or delay the onset of maladaptive correlates of aging in human beings. This journal is indexed on PubMed Central, MedLine, CAS, Scopus and the Elsevier
Bibliographic databases. The manuscript management system is completely online and includes a very quick and fair peer-review system, which is all easy to use. Visit http:/www.dovepress.com/ testimonials.php to read real quotes from published authors. 